\title{
Le club de football et la culture de masse. Notes sur un projet de recherche inédit de José Cutileiro
}

\author{
Nuno Domingos \\ Instituto de Ciências Sociais e Humanas - Universidade de Lisboa \\ nuno.domingos@ics.ul.pt
}

Victor Pereira

Université de Pau et des Pays de l'Adour

Victor.pereira@univ-pau.fr

\section{Résumé}

En 1965, José Cutileiro, alors doctorant en anthropologie sociale à l'Université d'Oxford, envoie à son directeur de thèse, John Campbell, l'ébauche d'un essai ethnographique portant sur l'équipe de football du Sport Lisboa e Benfica où il a mené une enquête pionnière. Cet essai n'arrive jamais à destination car il est intercepté et conservé par la police politique portugaise. Plus de cinq décennies après sa rédaction, ce texte est publié pour la première fois. Il révèle un programme de recherche et des problématiques qui restent encore pertinents non seulement pour l'étude du football au Portugal mais surtout pour l'interprétation des structures historiques de la société portugaise. La publication de ce document inédit est précédé par une présentation qui insère la recherche par José Cutileiro dans les courants de l'anthropologie de l'époque, le mettant notamment en rapport avec les recherches menées par certaines figures de l'école de Manchester comme Max Gluckman.

\section{Mots-clefs}

football - histoire de l'anthropologie - Estado Novo - nationalisme - Portugal

O Clube de futebol e a cultura popular moderna. Notas sobre um projeto de investigação de José Cutileiro 


\title{
Resumo
}

Em 1965, José Cutileiro, então doutorando em antropologia social na Universidade de Oxford, enviou ao seu orientador, John Campbell, o rascunho de um ensaio etnográfico sobre o Sport Lisboa e Benfica, clube de futebol português sobre o qual vinha a desenvolver uma investigação pioneira.

Este ensaio nunca chegou ao seu destino porque foi intercetado e guardado pela polícia política portuguesa. Mais de cinco décadas após a sua redação, publicamos pela primeira vez este texto, que revela um programa de pesquisa assente em questões que permanecem relevantes, não apenas para o estudo do futebol em Portugal, mas sobretudo para a interpretação das estruturas históricas da sociedade portuguesa. A publicação deste documento inédito é precedida por uma apresentação que integra a investigação realizada por José Cutileiro nas correntes da antropologia da época, relacionando-a nomeadamente com as pesquisas sobre futebol realizadas por algumas das maiores figuras da chamada escola de Manchester, caso de Max Gluckman.

\section{Palavras-chaves}

futebol - história da antropologia - Estado Novo - nacionalismo - Portugal

\section{The Football Club and Mass Culture. Notes on an Unpublished Research Project by José Cutileiro}

\begin{abstract}
In 1965, José Cutileiro, then a PhD candidate in social anthropology at the University of Oxford, sent to his supervisor, John Campbell, the draft of an ethnographic essay on the Portuguese football club Sport Lisboa e Benfica, a subject on which he was developing a pioneering research.

Intercepted and guarded by the Portuguese political police, this essay never reached its destination. More than five decades after its creation, we published for the first time a document that proposes a research program based on issues that remain relevant today, not only for the study of football in Portugal and elsewhere but especially for the interpretation of the historical structures of the Portuguese society.

The publication of this document is preceded by a study that relates the research carried out by José Cutileiro with the anthropological field of the time, focusing in
\end{abstract}


particular on the researches on football developed by some of the greater researchers of the Manchester School, namely Max Gluckman.

\section{Keywords}

football - history of anthropology - Estado Novo - nationalism - Portugal

Pendant la dictature de l'Estado Novo portugais (1933-1974), la police politique ne respectait pas le secret de la correspondance des individus et ouvrait, sans aucun contrôle judiciaire, les courriers qui lui paraissaient suspects ou qui lui semblaient constituer une source de renseignements (Pimentel 2007). Les lettres envoyées ou reçues de l'étranger constituaient une cible privilégiée pour les agents de la police politique qui prétendaient connaître l'action des exilés et craignaient les complots fomentés au-delà des frontières. Du travailleur émigré écrivant à sa famille, à la mère écrivant à son fils en exil au ministre ou haut fonctionnaire échangeant avec des proches, nul n'échappait à ce regard inquisiteur. Une fois lues - et traduites de langues étrangères le cas échéant le destin de ces correspondances variait. Elles pouvaient être remises dans le circuit postal, après avoir été photocopiées ou retranscrites lorsqu'elles contenaient des informations jugées pertinentes. Remettre la lettre dans le courrier pouvait permettre à la police politique de conserver une source de renseignements en ne suscitant pas la méfiance des interlocuteurs. Toutefois, dans certains cas, la police politique conservait tout simplement l'original du courrier, au risque d'alerter les correspondants et de les amener soit à cesser leurs échanges soit à utiliser des moyens pour contourner ces interceptions (utiliser des porteurs, employer un langage chiffré, l'envoyer à d'autres adresses). Le document que nous publions ci-après correspond à ce dernier cas de figure : envoyé du Portugal et destiné à la Grande-Bretagne, ce courrier a été intercepté par la police politique, traduit de l'anglais et conservé dans ses fichiers. Tant l'auteur du courrier - José Cutileiro, alors doctorant en anthropologie - que son destinataire - John Campbell, enseignant en anthropologie au St Antony's College de l'Université d'Oxford - n'ont pas su à l'époque que ce document avait été intercepté.

José Cutileiro ne l'a appris que 42 ans plus tard, lorsque nous l'avons contacté pour le questionner sur la recherche ethnographique qu'il avait menée en 1965 sur le club de football du Sport Lisboa e Benfica. Donnant les lignes majeures d'un article qu'il désirait publier en Grande-Bretagne, ce texte est donc 
resté pendant quatre décennies dans les archives et repose désormais à la Torre do Tombo. Si José Cutileiro a tiré un texte de cette ethnographie, publié dans la revue culturelle $O$ Tempo e o Modo (Cutileiro 1965), il n'y aborde pas exactement les mêmes points que ceux qu'il prétendait traiter dans la version anglaise. En effet, comme on pourrait s'y attendre, les dimensions politiques du football ne sont pas traitées ouvertement dans l'article du O Tempo e o Modo. Sachant qu'il serait lu - et éventuellement interdit - par la censure, l'auteur pratique l'auto-censure et multiplie les allusions et les sous-entendus, procédés communément employés par les écrivains et les journalistes sous l'Estado Novo.

José Cutileiro, né à Évora (Alentejo) en 1934, a commencé, en 1964, un doctorat en anthropologie sociale à l'université d'Oxford. Son parcours est loin d'être linéaire. Il suit d'abord des études d'architecture à l'École des Beaux-Arts de Lisbonne. Puis, comme son père, il mène des études de médecine tout en participant à la vie intellectuelle lisboète. Il écrit dans la revue Almanaque, fondée en 1959, à laquelle collaborent également des écrivains tels que José Cardoso Pires ou Alexandre O'Neill. En 1961, il publie un recueil de poésie chez l'éditeur Moraes dirigé par António Alçada Baptista, l'une des figures de proue de l'opposition catholique à l'Estado Novo. Il ne pratique pas la médecine et part à Oxford où il entreprend une thèse de doctorat sous la direction de John Campbell. Ce dernier, né en 1923, est enseignant à Oxford, après s'être également dirigé tardivement vers l'anthropologie. Ayant suivi des études d'économie à Cambridge, Campbell est mobilisé pendant la Seconde Guerre mondiale. Il combat en Afrique du Nord et en Italie où il est blessé. Fin 1944, il est envoyé, comme observateur, en Grèce où a éclaté une guerre civile. A son retour à la vie civile, il est atteint par la tuberculose. Grâce notamment à une rencontre avec Edward Evans-Pritchard, il se tourne vers l'anthropologie et s'intéresse à la Grèce qu'il a connue quelques années plus tôt, délaissant l'Afrique, l'Amérique latine ou le Pacifique, destinations alors privilégiées par les anthropologues. Il étudie des bergers nomades dans la région de l'Épire, les Saracatsanes".

La lettre envoyée par José Cutileiro à John Campbell relève d'un exercice particulier : la lettre d'un doctorant cherchant à rendre compte de ses avancées (ou non avancées) à son directeur. On perçoit que la relation tissée entre José Cutileiro et John Campbell n'est pas emprunte de la déférence et de l'asymétrie hiérarchique qui peut régir un tel rapport. Cutileiro n'est pas un jeune doctorant, il a déjà 31 ans. La différence d'âge est relativement faible - 11 ans - et les deux interlocuteurs partagent une trajectoire similaire: ils ont suivi d'autres

1 Sur la trajectoire de John Campbell, voir Smith (2008). 
études avant de commencer leur terrain en anthropologie (Campbell avait également 30 ans quand il a réalisé son terrain en Grèce).

Cutileiro expose à son directeur les difficultés matérielles qui l'ont empêché d'être sur son terrain, à Vila Velha ${ }^{2}$. Il a dû trouver un emploi qui l'a retenu à Lisbonne. Toutefois, dans la capitale, il s'emploie à trouver un terrain lui permettant de saisir les problématiques principales de ce que l'on a baptisé l' "anthropologie de la Méditerranée" : le clientélisme surtout, mais aussi l'honneur et la honte. La référence initiale au "patronage" n'est pas anodine. L'année précédente, Campbell a publié un ouvrage, issu de sa thèse, intitulé: Honour, Family and Patronage. A study of institutions and moral values in a Greek mountain community (Campbell 1964). Ce livre va constituer un classique de l'anthropologie méditerranéenne et inciter de nombreux doctorants à travailler avec John Campbell sur la Grèce mais aussi sur l'aire dite méditerranéenne ${ }^{3}$. Cutileiro choisit comme terrain un village de l'Alentejo, Vila Velha. Le choix de cette région est, en soi, novateur dans le champ, encore réduit, de l'anthropologie portugaise. Les anthropologues portugais privilégiaient surtout le nord du pays, marqué par une forte religiosité, des familles élargies et la prédominance de la petite exploitation familiale. Dans le Nord, le principal anthropologue au cours des années 1950, Jorge Dias, était à la quête des origines de l'identité nationale et des formes d'organisation communautaire considérées comme des résurgences d'une sorte de communisme primitif (Pina Cabral 1991: 22 et seq ; Leal 2007: 149 et seq). En étudiant l'Alentejo, Cutileiro met en exergue un autre Portugal : un Portugal méditerranéen par le climat et la production agricole (prédominance du blé et de l'olivier), un Portugal où l'Église a perdu une grande partie de ses fidèles et où dominent les familles nucléaires. Surtout, à la différence du nord du pays, il se penche sur une société fortement polarisée entre, d'un côté, grands propriétaires accumulant capital économique, social et politique et, d'un autre côté, journaliers vivant dans la pauvreté et la précarité, destitués de droits politiques et syndicaux. Dans son livre publié en Grande-Bretagne en 1971 (Cutileiro 1971) (et seulement après la révolution des CEillets au Portugal avec le titre Ricos e Pobres no Alentejo. Uma sociedade rural portuguesa [Cutileiro 1977]), Cutileiro ne porte pas sur le village qu'il étudie un regard idéalisé mais, au contraire, pointe du doigt les dures conditions de vie des travailleurs, les dominations dont ils souffrent, la méfiance qu'ils révèlent vis-à-vis de l'État et des plus riches.

2 Nous conservons le nom donné par José Cutileiro à ce village afin de préserver l'anonymat de ses habitants.

3 Voir le «mélange » rédigé par des étudiants de John Campbell : Mark Mazover (2008). 
Certains des questionnements qui sont au cœur de son travail sur Vila Velha se retrouvent dans l'esquisse ethnographique qu'il adresse à son directeur de thèse sur le Sport Lisboa e Benfica. Cette ethnographie - menée pendant 2-3 semaines selon ses souvenirs ${ }^{4}$ - sur le quotidien d'un club de football professionnel, s'attachant aux joueurs, aux entraîneurs, à l'encadrement médical, aux dirigeants mais aussi aux supporteurs est probablement l'une des premières réalisée non seulement au Portugal mais aussi internationalement. L'ouvrage de Hunter Davies (1972), qui a suivi pendant une année les coulisses du Tottenham Hotspur Football Club, est souvent considéré comme le premier du genre. Or il n'est publié qu'en 1972, sept ans après l'étude de Cutileiro.

En 1965, le football n'est toutefois pas un terrain totalement vierge pour l'anthropologie. Ces dernières années, des travaux ont mis en exergue l'importance du football comme objet de recherche de la dénommée « école de Manchester » d'anthropologie sociale et surtout le rôle pionnier joué par l'anthropologue sud-africain Max Gluckman (Gordon et Grundlingh 2016). Dans la lettre envoyée à John Campbell, quelques indices laissent supposer que Cutileiro connait les travaux de ses collègues de Manchester. Cutileiro avait pu lire le premier travail d'un anthropologue de Manchester qui, de manière systématique, avait analysé le football. Il s'agit du livre de Ronald Frankenberg, Village on the border. A Social Study of Religion, Politics and Football in a North Wales Community, publié en 1957 et issu d'une thèse dirigée par Gluckman (Frankenberg 1957). Gluckman, dans la préface, définit l'ouvrage comme une étude des activités qui soutiennent le désir des habitants d'un petit village gallois d'appartenir à leur communauté. Ces activités incluent une chorale, un brass band, une compagnie de théâtre, un carnaval et le club de football. Pour Gluckman, la monographie de Frankenberg a su tirer bénéfice des avancées les plus récentes de l'anthropologie sociale développée en Afrique, suggérant ainsi des lignes de comparaison qui nient l'existence de quelconques irréductibilités culturelles. L'analyse de Frankenberg - un marxiste influencé non seulement par les travaux qui, à partir d'autres aires disciplinaires, étudiaient la société britannique mais aussi par les recherches urbaines, comme celles du nord-américain William Footwhite ${ }^{5}$ - explique comment la construction de

4 Entretien avec José Cutileiro, Estoril, 24 août 2017.

5 Dans une nouvelle édition de 1990 (Frankenberg 1990), Frankenberg publie un nouveau texte - "Village on the border, a text revisited" - où il retrace son parcours biographique et intellectuel, faisant référence à un univers de références plus amples que celles présentes dans l'édition de 1957. Ces références incluent ses contemporains étudiant les rapports entre 
la communauté est dynamique et forgée dans le conflit, dans les luttes pour la notoriété et dans les différents espaces de la participation quotidienne. La façon dont les conflits sportifs contribuent à renforcer le sens communautaire, au lieu de le perturber, est une question qui intéresse Gluckman, dans le prolongement de ses travaux en Afrique (Gluckman 1954, 1955). En 1956, l'année précédant la publication du livre de Frankenberg, un sociologue américain d'origine allemande, Lewis Coser, publie un ouvrage, qui deviendra plus tard un classique, sur les fonctions sociales du conflit (Coser 1956). Dans la nouvelle édition de son ouvrage, Frankenberg fait référence à la proximité théorique de son travail, dans le prolongement des recherches de Gluckman, avec celui de Coser qu'il connaîtra cependant plus tard. Cutileiro, dans sa lettre à John Campbell, se révèle également sensible aux effets sociaux des conflits produits par les identités sportives. Mas comme nous le verrons plus tard, dans le contexte de l'Estado Novo, leurs interprétations vont suivre des voies distinctes.

Les publications académiques de Gluckman sur le football sont rares et certaines de ses analyses les plus importantes ont été diffusées dans des articles de journaux ou lors d'interventions radiophoniques. Comme le suggèrent Robert Gordon et Marizanne Grundlingh, l'intérêt de Gluckman pour ce sport découle de son expérience d'intégration à la ville de Manchester et à la société anglaise de manière plus générale. Son rapport au club de Manchester United aurait été décisif dans le succès de cette intégration. Ce qui a suscité l'intérêt de Gluckman pour ce sport est l'importance de la communauté formée par les supporters de l'United et du rituel de la marche vers le stade dans l'intégration d'un «étranger» dans un pays différent. Gordon et Grundlingh vont même plus loin : l'expérience communautaire d'Old Trafford, stade mythique de l'United où Gluckman amenait fréquemment ses étudiants pour assister aux rencontres, a été à l'origine des développements les plus structurants de l'anthropologie et notamment de l'évolution du concept de "communauté ». Un des étudiants qui accompagnait Gluckman au stade était Victor Tuner. Toujours selon Gordon et Grundlingh, l'élaboration théorique du concept de Communitas aurait également été influencée par la manière dont Gluckman, Turner et d'autres étudiants observaient la création d'une communauté émotionnelle qui, bien que principalement constituée d'un public d'hommes anglais d'origine ouvrière, se définissait en premier lieu par le lien au club, indépendamment de leur origine sociale, nationale, religieuse ou ethnique (Turner 1974, 2012). En ce sens, libéré d'un quelconque axe «primordialiste»,

changement social et continuité culturelle, comme Edward Palmer Thompson, Raymond Williams ou Richard Hoggart. 
ce collectif formait une communauté civique plus ouverte, basée sur un patriotisme footballistique. Comme l'a noté Gluckman

I watch Manchester United play Manchester City, and cheer for United with my fellow spectators, it does not matter that I have lived in Manchester only for a few years, that I am a Jew, that I come from South Africa, that I am a university teacher: I am at one with the host of other United supporters of different ethnic origin, of different religious persuasion, and of different occupation.

GLUCKMAN 1959

Cette expérience communautaire, qui provoquait chez Gluckman et Turner un état de transcendance, consolidait une singulière structure de parenté (Gordon \& Grundlingh 2016: 26). Pour Gordon et Grundlingh, la définition du concept de communitas avancée par Turner - "the transient personal experience of togetherness" - a pu être inspirée par le rituel du match à Old Trafford (idem. : 27). Gluckman reviendra plus tard sur la question du rapport entre identités sportives et conflit social et sur la manière dont ces identités contribuaient à cimenter les logiques de cohésion sociale (Gluckman 1973).

Le projet esquissé par José Cutileiro et les intérêts des anthropologues de Manchester ont des points communs. Ils semblent toutefois partir de cadres distincts. Gluckman et ses élèves cherchent à appliquer dans le contexte du nord industriel de l'Angleterre leurs avancées théoriques sur la communauté, le rituel et le conflit développées dans leurs terrains en Afrique. Cutileiro, de son côté, interprète le football à partir des principes d'une analyse issue de l'anthropologie méditerranéenne. À l'inverse des anthropologues de Manchester, Cutileiro prétend étudier le club et cherche à faire une ethnographie de ses dirigeants et de leurs relations avec les autres éléments du département de football, ayant recours aux analyses sur le patronage. À partir de ce point de départ, il désire saisir les effets plus vastes du football sur le public, dans un contexte politique dominé par une dictature. Gluckman, lui, préfère partir du public, avec lequel il partage l'immersion dans une communauté, pour évaluer la constitution d'une communauté civique, affective et démocratique.

Dans le texte de Cutileiro, l'importance du patronage comme moyen d'interprétation de certaines structures fondamentales de la société portugaise se note immédiatement. C'est grâce au patronage qu'il a pu entrer sur son terrain ethnographique. Introduit par le biais d'un ami de son père, jouissant d'un prestige social comme il le reconnait, Cutileiro peut s'immiscer dans une institution qui cultive le secret, se protège de la presse et des regards extérieurs. Comme le notent immanquablement les études sociologiques ou 
anthropologiques sur les joueurs de football professionnels, ces derniers sont difficilement accessibles, à la fois protégés par leur statut de star mais aussi conscients de leur capital culturel réduit (Roderick 2006, Beaud 2012, Rasera 2016). Ils rechignent ainsi à se confier à des chercheurs, acte dont ils ne tirent aucune rétribution (à la différence, parfois, des discussions avec les journalistes dans l'optique de leur valorisation sur le marché des transferts). Cutileiro réussit donc à enquêter dans un monde fermé et dont la fermeture s'est accrue depuis du fait de la multiplication d'acteurs (agents de joueur notamment que Cutileiro ne mentionne pas) et des ressources financières en jeu.

Plusieurs idées avancées dans ce texte convergent avec les résultats auxquels ont abouti, plusieurs années voire des décennies plus tard, les quelques chercheurs qui se sont penchés sur ce milieu professionnel particulier. Par exemple, Cutileiro met en exergue la vulnérabilité de ces joueurs soumis à une concurrence effrénée, craignant de se blesser ou d'être rejetés par l'entraîneur ou la direction du club. Comme l'a récemment souligné Frédéric Rasera, les footballeurs sont « insérés dans une organisation du travail qui est sélective et concurrentielle. Cette asymétrie entre le nombre de travailleurs sportifs présents au sein de l'effectif du groupe professionnel et le nombre de places disponibles pour la compétition place les joueurs dans un rapport de dépendance personnalisée au coach » (Rasera 2016 : 71). L'attention de Cutileiro aux asymétries sociales et, pour reprendre les concepts de Pierre Bourdieu, aux formes de la domination symbolique qu'il développe dans Ricos e Pobres no Alentejo est ici également présente. Il note qu'en dépit de leur statut de stars, les joueurs sont traités comme de simples employés, voire comme des enfants, soumis à un étroit contrôle quotidien, à une "domination rapprochée" (Memmi 2008) niant leur droit à une vie privée. Afin de mieux les discipliner (nourriture, sorties, sommeil), les joueurs célibataires sont logés par le club dans des locaux spartiates et n'ont pas la liberté de disposer d'eux-mêmes. Bien que talentueux et possédant un capital rare et valorisé, les joueurs sont dans la dépendance des dirigeants qui proviennent des élites sociales du pays, réunissant capital social, culturel, et pour certains d'entre eux, politique 6 . Béla Guttmann, entraineur d'origine hongroise qui gagna les deux (seules) coupes d'Europe remportées par le Sport Lisboa e Benfica (en 1961 et en 1962) se plaignait lui aussi d'être traité avec arrogance et dédain par les dirigeants du club qui ne reconnaissaient pas ses compétences professionnelles (Claussen 2015: 110-111).

Létude de Cutileiro représente le Benfica comme une institution totale, presque totalitaire, gérée dans le cadre d'un modèle de relations de patronage

6 Sur les dépendances dans lesquels se retrouvent les "génies", contraints de s'adapter aux goûts et volonté des dominants, voir le classique de Norbert Elias (1991). 
alors en changement. La grande interrogation de Cutileiro est de comprendre pourquoi, à l'inverse de nombreuses institutions d'un pays pauvre comme le Portugal, le Sport Lisboa e Benfica est devenu si efficace au niveau européen. La réponse semble résider dans les caractéristiques autoritaires du pays, marquées par le régime de patronage, combinées avec une dynamique institutionnelle de professionnalisme sportif qui se révèle alors inévitable, au regret de certains dirigeants du club qui préfèrent un modèle sportif basé sur les valeurs de l'éducation morale et du sportmanship amateur. Ces dirigeants opposés au primat du spectacle commercial n'ont pas réussi à freiner, selon Cutileiro, une évolution entrainée par les transformations économiques, par l'individualisme propre aux sociétés méditerranéennes et par l'intérêt du gouvernement à instrumentaliser ce sport à des fins politiques. Cette analyse des résistances des élites dirigeantes contre le sport de masses parait difficile à concilier avec une autre idée suggérée par Cutileiro : l'idée selon laquelle c'est la propre massification du spectacle sportif qui a offert une notoriété inédite à un ensemble de notables, aidant à transformer le modèle du patronage ${ }^{7}$.

L'hypothèse d'un club qui a atteint un succès sportif sortant de l'ordinaire en se fondant sur le professionnalisme autoritaire et sur l'exploitation d'une main-d'œuvre est, sans aucun doute, fort stimulante ${ }^{8}$. Cette hypothèse gagnerait à être confrontée aux archives des différents clubs portugais et à nourrir des recherches approfondies. Or, en 2018, de telles recherches n'existent toujours pas et la publication de cet inédit de Cutileiro espère inspirer de tels travaux.

Il faut toutefois interroger les limites de cette interprétation s'appuyant sur les postulats de l'anthropologie méditerranéenne, postulats qui ont fait l'objet de nombreuses critiques ces dernières années (Cabral 1991: 69-90, Bromberger et Durand 2004). Il serait utile de comparer le projet de recherche de Cutileiro avec d'autres cas de clubs, tant dans des sociétés méditerranéennes, dans le cadre de régimes dictatoriaux ou non, que dans les autres contextes où s'est développé un régime de professionnalisme plus développé. Sans écarter le présupposé de l'analyse sur ce professionnalisme autoritaire, où se distingue le rôle de certaines élites sociales dans un pays radicalement inégal comme le Portugal, il serait important de comprendre de quelle manière les échanges internationaux réalisés à l'intérieur d'un champ d'activité en grand

7 Idée qui apparaît de manière plus explicite dans son article publié dans O Tempo e o Modo (Cutileiro, $1965: 320$ ).

8 Il serait aussi important de saisir comment cette exploitation de leur force de travail a été vécue par les joueurs qui concevaient le football comme une manière de connaitre une mobilité sociale. Sur ces parcours et représentations, voir Cleveland (2017). 
développement ont permis le partage de techniques, tant les techniques sportives - des systèmes d'entrainement physique aux systèmes tactiques - que celles qui découlent de l'organisation complexe du travail dans un club de football ou celles qui passent par le contrôle de la vie des sportifs. Ce professionnalisme est entraîné par un processus relativement moderne et tendanciellement global que Norbert Elias a appelé le processus de "sportivisation" (Elias 1971 : 92). Il est évidemment injuste de confronter Cutileiro à un ensemble d'interprétations qui ont été développées après ses propositions d'analyses datant de 1965. Mais cette confrontation révèle la nécessité de prendre sa proposition d'interprétation d'un club de football portugais en pleine dictature comme un principe important pour la réalisation de nouvelles recherches. Nous considérons, du reste, que l'hypothèse du professionnalisme autoritaire et clientéliste avancée par Cutileiro peut s'appliquer avec profit au contexte du football portugais, notamment la gestion des clubs, après la chute de l'Estado Novo. L'étude de la circulation des élites entre les champs sportif, politique, au niveau central et local, et économique après le 25 avril 1974 révèle l'existence d'importantes continuités dans le système de notabilité clientéliste (Kumar 2012). Comme l'explique, de manière prémonitoire, Cutileiro dans son article publié dans $O$ Tempo e o Modo, la modernisation de ce modèle de patronage sportif va progressivement transformer le fonctionnement des clubs traditionnels d'associés (sócios), fondamentalement basé sur la participation des sócios et sur l'offre d'installations pour la pratique sportive. Sur ce type de fonctionnement, l'État reconnaissait les clubs comme des institutions d'utilité publique. Or, les clubs vont devenir des entreprises gérées dans une logique capitaliste, cherchant avant tout les profits nécessaires pour continuer à obtenir des victoires $(\mathrm{Cu}-$ tileiro $1965: 328)$.

\section{La question du nationalisme}

Dans ce texte, Cutileiro lance plusieurs pistes de recherche qu'il n'approfondira pas ultérieurement mais que l'on retrouve au cœur des questionnements des chercheurs en sciences sociales qui se sont penchés sur le football dans les décennies suivantes : les footballeurs comme travailleurs, la "passion" ressentie par les supporteurs pour cette "bagatelle la plus sérieuse du monde" (Bromberger 1998), la relation entre football et nationalisme.

Cette dernière piste étant au cœur de ce dossier de Lusotopie, nous nous permettons de la développer un peu dans le cas portugais. La place du football pendant l'Estado Novo a souvent été réduite au $3 \mathrm{~F}$ (Fado, Football, Fátima), réduction simplificatrice et fort peu opératoire. Dans le prolongement d'études 
se voulant critiques (Vassort 2005, Brohm et Perelman 2006), ce sport a fréquemment été présenté comme une sorte d' "opium du peuple", manipulé par la dictature pour maintenir la population dans l'apolitisme voire pour susciter l'adhésion populaire. Cependant, ces lieux communs ne s'appuient pas sur une recherche approfondie et les rares travaux qui ont étudié le football pendant l'Estado Novo nuancent cette vision. En effet, la dictature s'est longtemps méfiée de ce sport qui exaltait les passions de la population, creusaient des divisions (entre villes et quartiers notamment) au lieu de renforcer "l'union nationale" promue par le régime (Kumar 2017). Dans les années 1930 et 1940, afin de lutter contre la décadence de la « race portugaise », la politique sportive du régime privilégie les activités physiques jugées plus bénéfiques pour le corps (et l'esprit) des pratiquants, comme la gymnastique. Le football est construit comme néfaste pour le corps et sa pratique est interdite dans la rue, la police pourchassant les contrevenants (Domingos 2010, Vidal et Gonçalves 2011) et, jusqu'au début des années 1950, aux mineurs. De plus, l'État refuse, jusqu'en 1960, d'accepter la professionnalisation des joueurs, aux dépens de la compétitivité des clubs portugais et de la sélection nationale. À titre de comparaison, le statut de professionnel des joueurs de football est légalisé en 1885 en Angleterre (Correia 2018: 41), en 1929 en Espagne ou en 1932 en France. Salazar qui, à la différence d'un Mussolini, ne pratique pas de sport, affiche une distance face au football qui représente le monde urbain, les foules et la modernité dont il se méfie. Il se trouve parfois dans les tribunes lors de rencontres de la Seleç̧ão et reçoit les joueurs après des victoires internationales mais montre peu d'enthousiasme pour ce sport. Au début des années 1960, alors qu'un système légal de paris sportifs se met en place (le Totobola), il indique au ministre de la Santé qu'il est plus adéquat de verser une partie des recettes au

développement de la natation, de la gymnastique et des sports collectifs, dont la voile, au lieu d'utiliser cet argent (...) pour professionnaliser encore plus les sports où des milliers d'individus, sans aucune culture physique, vont assister à l'effort de douzaine d'athlètes déjà professionnels 9 .

Il reprend ainsi à son compte les critiques contre la professionnalisation du football formulées par les défenseurs d'une massification du sport amateur, massification destinée à améliorer l'hygiène de la population.

9 Lettre du ministère de la Santé et de l'Assistance adressée au président du Conseil, António de Oliveira Salazar, 31 mars 1961, Arquivo Nacional/Torre do Tombo, Arquivo Oliveira Salazar, AOS/CP-54. 
Une des dimensions les plus importantes du projet de recherche proposé par Cutileiro à son directeur de thèse est le rapport qu'il établit entre le club de football, comme instance de professionnalisme autoritaire gouverné par un modèle renouvelé de patronage, et le régime politique. Pour l'anthropologue portugais, le grand club de masses est un moyen, en grande partie nouveau, de diffusion d'un système de patronage massifié. Ainsi, il se réfère au rôle des dirigeants des clubs comme agents d'un processus d'aliénation, dans son sens marxiste. Il ajoute que l'organisation du football portugais offre aux masses un bouc émissaire pour les difficultés de la vie quotidienne, lorsque leur club perd, ou une drogue délicieuse, lorsque leur club gagne. Nous ne sommes pas loin de l'idée d'un opium du peuple. Cutileiro n'assume pas cette théorie sans la justifier théoriquement et méthodologiquement. C'est ici qu'il est utile de revenir aux approches de Gluckman et de ses étudiants à propos du match de football. Tous reconnaissent le rôle structurel du conflit et de la participation des individus dans des interactions ritualisées pour la construction de consensus sociaux. Mas alors que Gluckman exalte, à partir de son expérience de supporter de Manchester United, une communauté émotionnelle, démocratique, inclusive, non discriminatoire, Cutileiro considère la "participation mystique » des masses dans le stade comme un simulacre de participation civique. L'anthropologue portugais considère également que les victoires du Benfica transforment le club en étendard du nationalisme portugais, ce qui rejoint le traitement nationaliste conféré par la presse sportive aux succès du club $^{10}$. La presse sportive contribue ainsi, comme João Nunes Coelho le signale des décennies après Cutileiro, à la dissémination de formes d'un nationalisme banal (Coelho 2012).

Au moment où Cutileiro rédige son texte, le Benfica connaît un âge d'or. Depuis 1961, le club a participé à quatre finales européennes, en remporte deux, et est l'un des plus grands clubs au niveau international. Ces succès tranchent dans ce pays rural, pauvre, et soumis à une dictature depuis près de 40 ans. Ils contrastent aussi avec le passé sportif du football portugais. Jusqu'alors les clubs portugais et la Seleç̧ão subissaient plutôt de lourdes défaites. Dans les années 1940, craignant que les résultats sportifs soient vus comme un baromètre du développement économique et social du pays, face à ces défaites

10 Cutileiro considère la presse sportive comme un univers possédant une dimension technique réduite, se limitant, fondamentalement, à être un moyen de dissémination de métaphores nationalistes et d'appels à l'honneur. Cette analyse marginalise les luttes internes au champ des journaux et les effets de la censure. Elle occulte également le rôle de la presse dans la promotion des rivalités inhérentes au projet de "sportivisation", rivalités qui dépassent les manières de voir nationales (et nationalistes). Voir Domingos et Kumar (2011). 
qui suggèrent le retard voire la décadence du Portugal, les autorités réduisent strictement les déplacements de clubs de football à l'étranger ou la venue de clubs étrangers (Pereira 2016). Il s'agit d'éviter des humiliations sportives qui flétriraient l'honneur national et viendraient remettre en cause la supposée œuvre de restauration mise en place par Salazar.

Il est toutefois vrai que la dictature a tenté de tirer profit des excellents résultats internationaux du Sport Lisboa e Benfica au début des années 1960. Outre ces victoires, c'est l'émergence au plus haut niveau de joueurs provenant de l'Outre-Mer - dont Eusébio, né au Mozambique - qui fait l'objet d'appropriations. La composition pluri-ethnique du Benfica (et de l'équipe nationale) tombe à point nommé alors que les guerres coloniales éclatent en Angola en 1961 (puis en Guinée-Bissau en 1963 et au Mozambique en 1964). L'équipe du Benfica est censée illustrer la force et la cohésion d'un pays pluri-continental et pluri-racial. Le Benfica est approprié dans de nombreux discours pour diffuser un "lusotropicalisme banal" (Cardão 2015) qui, grâce à la presse, la radio et la télévision, entrent dans la plupart des foyers portugais. La figure d'Eusébio est récupérée par une multitude d'acteurs qui font de lui le représentant des populations africaines refusant l'indépendance des colonies et luttant pour le maintien d'un Portugal du Minho à Timor (Domingos 2014, Cardão 2014). Eusébio défend l'unité du Portugal sur les terrains tandis que des milliers d'Africains le font au sein de l'armée portugaise, où ils sont enrôlés. Dans son texte, Cutileiro montre bien comment des Portugais vivant loin de Lisbonne, n'ayant parfois jamais été au Estádio da Luz, vivent avec passion les rencontres de leur club. Cette grande communauté de personnes qui ne se connaissent pas les uns les autres est seulement possible grâce aux médias qui fournissent aux individus surtout les hommes - un "stock social de connaissance" (Domingos 2017) qu'ils mobilisent ponctue leurs interactions quotidiennes.

Toutefois, l'absence dans le projet de Cutileiro d'une ethnographie des tribunes du Estádio da Luz, qui rapprocherait l'anthropologue des supporters, laisse en suspens les questions sur la linéarité de ce processus d'aliénation politique. En créant des moyens d'identification et de partage des grammaires sociales d'interaction, les grands clubs de football se distinguent également en fournissant des capitaux essentiels pour la vie quotidienne dans les villes modernes. Et, en ce sens, la participation sociale canalisée par le football devrait être pensée au-delà de la "participation mystique" dans le stade. En réalité, un commentaire similaire pourrait être formulé à l'encontre de l'analyse de Gluckman. Les deux anthropologues attribuent au rapport du supporter à son club une dimension mystique, typique d'une expérience religieuse. Mais s'ils sont d'accords sur ce point, leurs interprétations divergent ensuite. Cutileiro, dans le Portugal de Salazar, relie ce rapport au club avec l'instrumentalisation 
politique et avec la célébration des valeurs nationales exaltées par la dictature. Dix ans auparavant, dans la Manchester industrielle, Gluckman célèbre le rapport au club comme un projet de camaraderie, sans frontières, l'esquisse d'une nouvelle communauté émotionnelle et de partages. Quand il parlait de communauté imaginée, Benedict Anderson tentait d'articuler la dimension mystique de la Communitas avec le projet d'un nationalisme civique, présent dans les processus d'indépendance américains. Nous en sommes assez loin, évidemment, dans la situation portugaise des années 1960. Lintérêt à comprendre les dynamiques propres au champ sportif, cherchant à interpréter le rapport des supporters à leur club sans que leurs actions, désirs et interprétations soient réduits aux effets d'un opium sportif, un intérêt qui constitue une des plus grandes conquêtes des études sur le sport, ne doit pas non plus écarter le débat sur l'influence du football dans l'espace public, influence qui varie significativement dans le temps et l'espace.

Plus de $5^{0}$ ans après sa rédaction, ce texte, pionnier sur de nombreux aspects et offrant de fertiles problématiques, mérite donc de sortir du dossier de la police politique dans lequel il a été rangé. D'autant plus qu'à l'exception de l'article publié dans O Tempo e o Modo, José Cutileiro n'a plus abordé le football dans son œuvre anthropologique. Dans les années qui suivent, Cutileiro termine sa thèse qu'il publie en 1971. La même année, il traduit en portugais un ouvrage majeur de l'anthropologie de la Méditerranée, dirigé par John Peristiany (1971). Entre 1971 et 1974, il enseigne quelques années l'anthropologie à la London School of Academics. Après la révolution des CEillets, il bifurque sur une carrière diplomatique, où il atteint de hautes fonctions dans des organisations internationales, et abandonne totalement l'anthropologie ${ }^{11}$.

\section{"Dear John,}

I'm trying to get used to this mechanical way of writing, not very successfully. This month has been a poor one (February) as far as fieldwork goes and I fell a bit frustrated. I went only three times to Vila Velha and for short periods. Lack of funds forced me to accept a temporary task working on the planification of some sections of a Portuguese exhibition in Brazil. It is almost

\footnotetext{
11 Sur sa carrière diplomatique, voir Cutileiro (2017).

12 L'original de cette lettre - ainsi qu'une traduction en portugais - se trouve aux Archives nationales de Lisbonne (Torre do Tombo). Arquivo PIDE/DGS, SC CI (2) 9538, NT 7566.
} 
over now and the pay will be free of doing anything but Vila Velha in the next few months.

As a sort of exercise since I had to stay in Lisbon for longer periods I made a short study of Benfica (the soccer club) and it turned up to be a very illuminating experience as a cross-analysis of some Portuguese institutions, much more in fact that bullfighting to which I had devoted some time in the summer. First: the way I got introduced. Through patronage. Although the players and managers are professionals, director and trustees are still amateurs. They are elected annually by and among the "sócios" (i.e. people who have membership of the club paying a monthly contribution of $20 \mathrm{esc}-5$; they number now more than 50000 ) and they tend to be in the last few years picked up among important personalities in Portuguese life: well-known doctors or lawyers, big business men, high rank service men, millionaires, etc. They are gradually outnumbering in the directions of the big clubs (mainly in Benfica) the old traditional type of directors - devoted amateur sportsmen, ex-amateur players, old fans of the club for the club's sake. The new type are mainly after personal prestige (which they get) or sometimes they are eminent persons chosen to "counter" the cult-of-personality chaps. All of them regardless of their motives get more than what they give and they do give time, ability, sometimes money. They get, in a country where there is no active political life, in the european democratic sense and no syndical (sic) activity as well, the next best to hit at a national level with less risk and a fuller sense of personal realization than any underground or half-underground opposition leader with the possible exception of the communist minority whose mystique provides them with a feed-back system that unables them to live out of their own failures. I should consider these club directors as typical cases of alienation in the Marxist sense.

Now I think I must tell you something more about soccer in Portugal, otherwise you would think I am exaggerating. Soccer is the big sport and the big show. Besides the thousands of "sócios" of the hundreds of clubs there are the further thousands or millions who are not paying members but feel strongly about the matter. There is, mainly in the provinces, a regional pattern of affiliation and as soon as a place is big enough for it to get two clubs which are rivals - the one from one side of the river or from the upper town and viceversa. But many times the clubs actualise or are supposed to actualise important local tensions: peasants and workers and mainly the rich and the poor. It is in its way a nice segmentary system - when you get up on the leagues local differences are blurred but the opposition to other regions. On the other hand a big dichotomy towers the system: the two big clubs Benfica and Sporting both from Lisbon. If you are from Lisbon yourself one of them is almost surely your first choice (I am Benfica, as a matter of fact. Middly). But the whole 
country divides itself over this issue, which goes along with local and regional preferences (parallel to). I sometimes think you could define a Portuguese as someone who is Benfica and hence anti-sporting or alternatively Sporting and hence anti-Benfica. People argue, quarrel, fight and eventually kill each others over the respective merits of the two Clubs. This final dichotomy subsides however in an international context: Benfica winning twice the European Cup and being in its way to a possible third victory identifies itself (or rather identified) not only with its ordinary addicted but also with every "true portuguese".

Now Benfica is one of the very few highly efficient Portuguese organisations of any kind and surely the more conspicuous one to the average citizen. In a country where the administration is slow and corrupted, the National Health Service and the educational institutions from primary schools to the universities are bogus, industry is in its teens, art, literature and music of scarcely any interest even for us, to become the best soccer club in Europe is acknowledged as a national glory. Only Salazar and Our Lady of Fatima have eventually been as efficient. Even in sports ours records are extremely poor. We never won an olympic medal in athletics, and performances in other activities are low - we usually loose international contests by great margins (with the possible exception of some forms of sailing). I was curious about this Benfica efficiency. And I decided to enquire on it.

This is where patronage comes in. One of the big directors of the Club this year is a professor of medicine and was a friend of my late father. I talked with him, he was sympathetic towards the idea and recommended me to the soccer section. Being a "senhor doutor" myself, i.e. a gentleman with a degree, the thing was even easier. And I got more introduced and intimate with the soccer section of Benfica (there are lots of others from chess to cross-country) than any newspaperman has ever been - and there are three sport newspapers in Lisbon with high circulation and almost entirely devoted to soccer. Because I was so to say patronized and camae myself from the patronizing group I was even allowed to break rules of secrecy of the club. This needs some explanation.

The soccer section is the vital part of the club. From it comes money, prestige, the club's raison dêtre for the majority of its socios. It has a director, a vice-director, doctors, male nursees, "masseurs", one head manager (a FrenchHungarian, the only foreigner ${ }^{13}$ ) two other managers, and about 40 professional players (juniors i.e. less than 18 non included). The players are well-paid by

13 Il s'agit d'Alexander (Elek) Schwartz, né en 1908 et décédé en 200o. Il a joué en Roumanie puis, à partir de 1934, en France. En 1940 il devient entraîneur en France. Il connaît dès 1955 une carrière internationale, travaillant en Allemagne, au Pays-Bas (dont il est le sélectionneur en 1964) et au Portugal (il entraîne Benfica en 1965 et Porto en 1970). 
Portuguese standards for people of their social groups of origin (they come from the working class or the lower middle-class when they were not "natives" from Portuguese Overseas) have an almost spartanian life. Those who are bachelors live in "Benfica's home" a big house in the outskirts of Lisbon with the outdoors and indoors looking of a second class provincial boarding house. They are subject to residence regulations stricter than those of an Oxford undergraduate ${ }^{14}$ and even in holiday they cannot, for instance, travel by plane. The married ones live in their homes but their personal life is controlled by the club almost as strictly as that of the bachelors. Moreover from Thursday to Sunday evening (games are usually at Sunday's afternoon) they are "concentrated" (the jargon expression) in the Home. The first eleven plays in the first league, the second in a special Lisbon tournament for Second elevens. They get different pays and "game prizes" accordingly and since Benfica has bought much more first class players than its first eleven needs this is a cause of friction and neurosis. Almost all of them could be considered as neurotic as one should expect from their living conditions. Some of these men are national idols and one at least (Eusébio) is internationally notorious. But when you meet them in their own environment you notice that they are little more than gladiators towards the gentlemen of the direction: they are addressed by their christian names and answer back respectfully using some of the more elaborated Portuguese formulae to accentuate social distance (mister). Although the best of them are able to amass a considerable quantity of money they know that at 35 they will be finished and that social climbing is altogether impossible (as against what happens with first-class Spanish bullfighters). Anything comparable with the knighthood of Matthews ${ }^{15}$ would be unthinkable in the Portuguese situation. Some of them are intelligent some are not, some read a lot some don't but all of them are much more aware of the contradictions of their own position and of the big alienation of soccer in Portugal than the thousands of fans who shout in the stadiums every Sunday afternoon and the majority of the directors and the sports newspapermen. The latter by the way are one the big instruments of this alienation: sportive press which in fact means soccer press is much more lively and relevant to the reality they are concerned with then ordinary Portuguese press. And outlet again for polemical and political urges: a game in its way mocking civic participation in a form not only allowed but encouraged

\footnotetext{
14 No female visitors.

15 Il s'agit d'une référence au joueur anglais Stanley Matthews (1915-2000). Matthews fut un des joueurs anglais les plus célèbres, avec une carrière exceptionnellement longue (de 1932 à 1965). Il remporta le premier ballon d'or (récompensant le meilleur joueur européen) en 1956 et fut anobli par la reine Elisabeth II en 1965 comme l'évoque José Cutileiro. Voir Tony Mason (1994).
} 
by the authorities. From a literary point of view they are of a particular type of style; they are not technical papers: word like honour, rage, race, shame and literary metaphors of half-cultivated ridiculous taste crop in frequently. In a recent match against Real Madrid, won 5-1 by Benfica, a goal by Eusébio was compared in one of these newspapers to the battle of Aljubarrota (1385) in which the Portuguese secured independence from Spain. ${ }^{16}$ The leading title said that Valle de los Caidos (valley of the fallen, national Spanish cemetery for those killed in the civil war) had moved to the Stadium where the match was held. This was however too much. The Spanish embassy protested and the Portuguese government thought it unbecoming to a political friendship they are eager to secure. The newspaper was suspended and the author of the article fired.

Back to the soccer section of Benfica, my precise field. They form in its way a closed society. One feels that some directors would like it to be a Spartan or Hitlerian organisation only with bachelors, a body of available prostitutes, and a clear euthanasian way of getting rid of the too old and the troublesome. This would be in a latin backward country perhaps the best way of achieving continuous victories in the European Cup, their avowed goal. On the other hand you find those directors who deplore professionalism who would like the club to come back to its old function of promoting the practice of sports by their own sake, with larger participation and less "showbusiness". But they cannot help. Economic general conditions, cultural traditions with emphasis in the catholic abhorrence for nudity and for the body and bodily activities, but including also the strong individualistic bias that you know so well in the mediterranean societies [ajout à la marge: and an awareness of oneself and the other that makes the fear of being ridiculous a strong social sanction] are against them. Against them too in the turn of events (highly patronized by the government and its supporters) that transformed Portuguese soccer into both a scapegoat for the hardships of life (if your club looses) and a short of delightful drug (if it wins). A form of shall we say mystical participation - it is not infrequent that people die of heart disease witnessing matches. The last one remember, two weeks ago, was seeing a match on the TV. He was described in the papers as a "true Benfica man" and a true sportsman. All this and also the emotions of the club life itself. Protesting loudly against decisions of the direction, writing letters to the manager disagreeding with him, shouting their concern and discontent when they fell like it. As the manager told me the other day: "ces types qui ne protestent jamais de rien, sauf les dimanches après-midi qu'ils viennent ici crier comme des fous".

16 Ce match a eu lieu le 24 février 1965. 
They are still two important points but I am not going to bother you with them now. The first one is the analysis of the mechanism of winning itself and of the social factors affecting the performances of individual players and of the team. I have some information and ideas about it which I will complete soon and submit to you. The second one is the collection of life stories of the players which I am undertaking. They form the trus inside view and the precise flesh where some of our concepts of this society take shape.

I would not take the risk of having bored to death with Benfica (besides the fact that it comes as a diversion from Vila Velha) but for two reasons. First as I forcibly to stay longer periods in Lisbon I wonder whether you think this may have been a useful exercise. Second: if so, should I enlarge it to 10 ooo correct English words and try the Hocart prize? ${ }^{17}$ Hocart of course would have begun it in a very different way "why should 22 men kicking a leather ball in a green field attract so many thousands to see it? The religious books of India ... etc.". Fascinating as it is I cannot at least for the time being cope with it that way.

Please let me know what you do think about this all. As I said in the beginning of this letter I will very soon be free to concentrate in Vila Velha again. My only consolation for this delay is that on the whole I have already been there longer than E.-P. ${ }^{18}$ has ever been with the Nuer. But is anyone preparing a thesis who has not felt the same?

Please give my best regards for your wife, to Sarah and Fifi. I miss 22, Winchester Road on the whole very much sometimes. If I have any up in my financial situation I am thinking of going to Oxford for a week in June to discuss with you the material that I will already have by the time.

$9 / 3 / 1965$

\section{Bibliographie}

Beaud, S. 2011, Traîtres à la nation? Un autre regard sur la grève des Bleus en Afrique du sud, Paris, La Découverte.

Brohm, J-M. et Perelman, M. 2006, Le football, une peste émotionnelle. La barbarie des stades, Paris, Gallimard.

17 Le Prix Arthur Maurice Hocart, créé en 1948 par Madame E. G. Hocart en mémoire de son mari, récompense le meilleur essai réalisé par un étudiant, quelle que soit sa nationalité, inscrit en anthropologie dans une institution britannique ou irlandaise.

18 Il s'agit d'un clin d'œil à Edward Evan Evans-Pritchard et de son étude sur les Nuer, en Afrique de l'Est. 
Bromberger, C. 1998, Football. La bagatelle la plus sérieuse du monde, Paris, Bayard.

Bromberger, C. et Durand, J.-Y. 2001 "Faut-il jeter la Méditerranée avec l'eau du bain", in D. Albera, A. Blok et C. Bromberger eds., L'anthropologie de la Méditerranée, Paris, Maisonneuve et Larose/Maison méditerranéenne des sciences de l'homme.

Cabral, J. de Pina 1991, Os contextos da antropologia, Lisboa, Difel.

Campbell, J. 1964, Honour, Family and Patronage. A study of institutions and moral values in a Greek mountain community, Oxford, Clarendon Press.

Cardão, M. 2014, "Um significante instrumental: Eusébio e a banalização do lusotropicalismo na década de 196o", in V. A. de Melo, F. de F. Peres, and M. Drumond eds., Esporte, Cultura, nação, Estado. Brasil e Portugal, Rio de Janeiro, 7 Letras : 172-188.

Cardão, M. 2015, Fado tropical. O luso-tropicalismo na cultura de massas (1960-1974), Lisboa, Unipop.

Claussen, D. 2015, Bela Guttmann, Uma história mundial do futebol, Lisboa, Paquiderme.

Coelho, J. N. 2002, Portugal, a equipa de todos nós: Nacionalismo, futebol e media, Porto, Afrontamento.

Correia, M. 2018, Une histoire populaire du football, Paris, La Découverte.

Coser, L. 1956, The Functions of Social Conflict, London, Routledge and Kegan Paul.

Cutileiro, J. 1965, "Os superportugueses: algumas notas sobre o Sport Lisboa e Benfica", O Tempo e o Modo, 25-26 : 319-330.

Cutileiro, J. 1971, A Portuguese Rural Society, London, Oxford University Press.

Cutileiro, J. 1977, Ricos e Pobres no Alentejo. Uma sociedade rural portuguesa, Lisboa, Sá da Costa.

Cutileiro, J. 2017, Abril e outras transições, Lisboa, Dom Quixote.

Davies, H. 1972, The Glory Game, London, Weidenfield \& Nicolson.

Domingos, N. 2010, "Building a motor habitus: Physical education in the Portuguese Estado Novo", International Review for the Sociology of Sport, 45 (1) : 23-37.

Domingos, N. et Kumar, R. 2011, "A grande narrativa desportiva: o desporto nos média em Portugal”, in J. Neves et N. Domingos eds., Uma história do desporto em Portugal, vol. I, Corpo, espaços e média, Vila do Conde, Quidnovi : 207-310.

Domingos, N. 2014, "O lugar de Eusébio na 'grande sociedade portuguesa", in V. A. de Melo, F. de F. Peres, and M. Drumond eds., Esporte, Cultura, nação, Estado. Brasil e Portugal, Rio de Janeiro, 7 Letras : 156-171.

Domingos, N. 2017, Football and colonialism. Body and Popular Culture in Urban Lourenço Marques, Athens, Ohio University Press.

Elias, N. 1971, "The Genesis of Sport as a Sociological Problem", in E. Dunning ed., The Sociology of Sport. A selection of readings, London, Frank Cass : 88-115.

Elias, N. 1991, Mozart, sociologie d'un génie, Paris, Seuil.

Frankenberg, R. 1957, Village on the Border, a Social Study of Religion, Politics and Football in a North Wales Community, London, Cohen and West. 
Frankenberg, R. 1990, Village on the Border, a Social Study of Religion, Politics and Football in a North Wales Community, Prospect Heights, Waveland Press.

Gluckman, M. 1954, Rituals of Rebellion in South-East Africa, Manchester, Manchester University Press.

Gluckman, M. 1955, Custom and Conflict in Africa, Oxford, Basil Blackwell.

Gluckman, M. 1959, “How Foreign Are You?”, The Listener, 15 Janvier 1959 : 99-102.

Gluckman, M. 1973, "Sport and Conflict", in O. Grupe, D. Kruz et J. Teipel eds., Sport in the Modern World - Chances and Problems, Berlin, Springer : 48-54.

Gordon, R. et Grundlingh, M. 2016, "Going for the reds: Max Gluckman and the Anthropology of Football", in A. Schwell, et ali. eds. New Ethnographies of Football in Europe: People, Passion, Politics, Basingstoke, Palgrave : 21-35.

Kumar, R. 2012, "Futebol e política no Portugal democrático: a lógica de conversão de capitais", in N. C. Tiesler et N. Domingos eds., Futebol Português. Política, género e movimento, Porto, Afrontamento : 83-114.

Kumar, R. 2017, A pureza perdida do desporto. Futebol no Estado Novo, Lisboa, Paquiderme.

Leal, J. 2006, Antropologia em Portugal. Mestres, percursos, transições, Lisboa, Livros Horizonte.

Mason, T. 1994, "Stanley Matthews, la genèse d'un symbole", Actes de la Recherche en Sciences Sociales, 103: 62-69.

Memmi, D. 2008, "Mai 68 ou la crise de la domination rapprochée", in D. Damamme et alii eds. Mai-juin 68, Les Éditions de l'Atelier : 35-46.

Pereira, V. 2016, "La Selecção portugaise, entre dictature, propagande coloniale et horizon européen", in F. Archambault, S. Beaud et W. Gasparini eds., Le football des nations. Des terrains de jeu aux communautés imaginées, Paris, Publications de la Sorbonne : $5^{1-74}$.

Peristiany, J. ed. 1971, Honra e vergonha. Valores das sociedades mediterrâncias, Lisboa, Fundação Calouste Gulbenkian.

Pimentel, I. F. 2007, A história da PIDE, Lisboa, Temas e debates/Círculo de Leitores.

Rasera, F. 2016, Des footballeurs au travail. Au cour d'un club professionnel, Marseille, Agone.

Roderick, M. 2006, The work of professional football. A labour of love, London, Routledge. Smith, M. L. 2008, "John Campbell", in M. Mazower ed., Networks power in Modern Greece. Essays in Honor of John Campbell, New York, Columbia University Press: 255-268.

Stewart, C. 2004, "João de Pina Cabral : Quelques réflexions d'Oxford", Recherches en anthropologie au Portugal, 10 : 63-66.

Turner, V. 1974, Dramas, Fields, and Metaphors. Symbolic Action in Human Society, Cornell University Press. 
Turner, V. 2012, Communitas: The Anthropology of Collective Joy, New York, Palgrave Macmillan.

Vassort, P. 2005, Football et politique: sociologie historique d'une domination, Paris, L'Harmattan.

Vidal, F. et Gonçalves, G. R. 2011, "O desporto na rua em Lisboa no início do século XX", in J. Neves et N. Domingos eds., Uma história do desporto em Portugal, vol. I, Corpo, espaços e média, Vila do Conde, Quidnovi : 147-166. 